irradiated sporozoites. In the 1980s, a group at New York University, showed that monoclonal antibodies against the circumsporozoite protein of rodent and human malaria protected against infection. They later cloned the gene coding for a CS protein, although vaccines based on this failed in trials because they did not elicit high titres of antibody.

Such 'sporozoite' (or pre-erythrocytic) vaccines are just one of three broad vaccine approaches each targeted at one of the three main phases of the parasite's lifecycle. Sporozoites, the form of the parasite injected by the feeding female mosquito, migrate through the bloodstream to the liver within half an hour. In the hepatocytes, they pass through several intermediate stages, and multiply, before emerging around a week later as tens of thousands of 'merozoites' (events up to this point are known as the pre-erythrocytic phase, which is also asymptomatic).

These merozoites invade red blood cells where they complete their cycle, and later rupture the cells and invade new red blood cells (the asexual erythroctye phase). The 'sexual phase' begins when some of these then form gametocytes - these are sucked up by feeding mosquitos, inside which they combine to form new sporozoites.

Pre-erythrocytic vaccines target the sporozoites themselves or the forms inside liver cells (although the parasites are inside the cell they express epitopes on its surface which can be detected by T-cells that kill the cells directly or by releasing lethal cytokines such as gamma interferon). The liver stages are in principle ideal targets because they remain in the cells for several days. The main difficulty is

\title{
Transgenic mosquitoes: a new solution?
}

Many scientists believe the time has come for a serious research effort to develop new techniques of vector control. In particular, rather than focussing on eradicating the mosquito, which is notoriously difficult to control, they are now focusing on creating transgenic insects incapable of transmitting malaria, and finding ways to spread the genes involved in natural resistance throughout wild populations.

Such genes are currently being mapped by genetic linkage studies of malariaresistant strains of mosquitoes. For example, several such genes have been mapped in a refractory strain of Anopheles gambiae by Frank Collins, a vector biologist at the Centers for Disease Control and Prevention and his collaborators Liangbiao Zheng and Fotis Kafatos at the European Molecular Biology Laboratory in Heidelberg.

Collins and Kafatos have collaborated in a multilaboratory effort to map the mosquito genome, which began in the late 1980 s with support from the US MacArthur Foundation and Britain's Wellcome Trust.

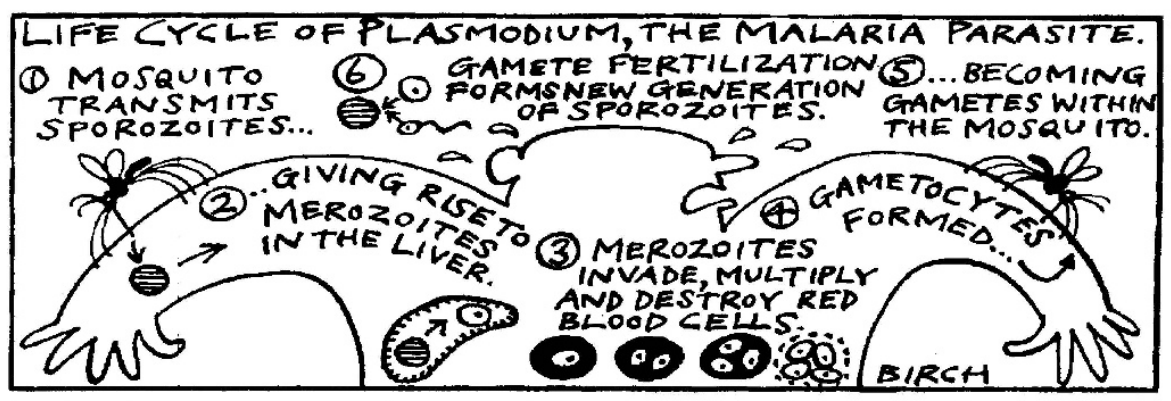

that there is as yet no way of making a vaccine which elicits a T-cell immune response.

'Anti-asexual blood stage' vaccines target the phase where merozoites infect blood cells - the phase that causes disease symptoms. One of the most studied vaccines is based on a surface protein of merozoites (MSP1), which gives protection in rodents and primate models - this protein is particularly promising because the regions involved in protection shows no variation. In contrast, another target, EMP-1 (or erythrocyte membrane protein) a parasite protein expressed on the surface of the red blood cell - and which is involved in cerebral malaria - shows high antigenic variation and is not suitable for vaccine development.

\section{Attack on parasite inside mosquito}

'Transmission blocking vaccines' interrupt fusion and development of parasite gametes in the mosquito itself. They do not protect individuals directly, but are aimed at reducing transmission, which is why they are sometimes called 'altruistic' vaccines. A fourth approach is therapeutic vaccines targetted against toxins released by the parasites

Another approach is to engineer mosquitoes carrying foreign genes that confer protection against infection. Julian Crampton anmd his colleagues at the Liverpool School of Tropical Medicine in Britain have cloned a mammalian gene for an antibody that attacks an antigen on the ookinete phase of the parasite in the mosquito.

The bigger challenge perhaps is to drive such engineered traits into the wild population. Work on Drosophila has shown that engineered mobile portions of genes or transposons - can integrate and be replicated in the genome of germline cells and spread quickly through the population. Releasing of a small number of transformed mosquitoes should result in the desired genes spreading throughout the wild population.

No one has transformed mosquitoes in a stable fashion using transposons. But researchers are optimistic that they are close to reaching this goal, while remaining aware that many safety issues need to be resolved before genetically engineered mosquitoes can be safely released into the environment. themselves or during red cell rupture.

Hopes for an anti-asexual blood stage vaccine were raised with a report by Colombian biochemist Manuel Patarroyo of a promising small trial of the controversial synthetic SPf66 vaccine (see Nature 332, 158; 1988). Early trials have been described as giving efficacies as high as 80 per cent, but their design has been criticized, and subsequent trials gave efficacies of just 31 and 8 per cent. One last year showed the vaccine had no effect. Patarroyo plans to test his SPf66 vaccine in 50 volunteers using the QS21 adjuvant in Colombia this summer.

In the 1990s, trials of three pre-erythrocytic vaccines in Africa and Asia have also yielded poor results, again because they were poorly immunogenic. A multistage vaccine, NYVAC-Pf7 developed by Virogenetics and Connaught, which showed promise in animal studies, has also given disappointing results. Such multistage vaccines make a concerted attack on several stages of the parasite within the body.

DNA vaccines are another novel approach. Stephen Hoffman, head of malaria research at the US Naval Research Institute in Bethesda, Maryland, plans to test a singlegene vaccine in volunteers this summer. If all goes well, the institute will then test a more sophisticated vaccine devised by Vical, a biotechnology company based in San Diego. The Italian subsidiary of the US company Chiron Vaccines is also working on a multistage DNA vaccine.

Apart from the promise of adjuvants, optimism is also high about CS-based vaccines because of the fact that insecticide-impregnated bednets have recently been shown to bring about large reductions in mortality. This suggest that the number of incoming sporozoites is a critical factor in mortality and that even a $\mathrm{CS}$ vaccine which did not give complete protection could have a big impact on deaths.

But one caveat in the study by SmithKline Beecham and the Walter Reed Army Institute is that the cut-off point used to define protection was 60 days, and a question remains whether protection will be sustained. A vaccine giving a short-lived effect might protect travellers but not people living in endemic regions. Another question is whether the adjuvants would be toxic in young children. Answers to these and other questions should come from vaccine trials under way in the Gambia. 